\title{
Decreased LRIG1 in fulvestrant-treated luminal breast cancer cells permits ErbB3 upregulation and increased growth
}

\author{
Meghan M. Morrison ${ }^{1}$, Michelle M. Williams ${ }^{1}$, David B. Vaught ${ }^{1}$, Donna Hicks ${ }^{1}$, Jiyeon Lim ${ }^{1}$, \\ Courtney McKernan ${ }^{1}$, Luigi Aurisicchio, Gilberto Cennaro, Catalina Simion, Colleen \\ Sweeney, and Rebecca S. Cook ${ }^{1,4}$ \\ ${ }^{1}$ Department of Cancer Biology, Vanderbilt University School of Medicine, Nashville, TN 27232 \\ ${ }^{4}$ Vanderbilt Ingram Cancer Center, Nashville, TN 37232
}

\section{Abstract}

ErbB3, a member of the ErbB family of receptor tyrosine kinases, is a potent activator of phosphatidyl inositol-3 kinase (PI3K) and mTOR signaling, driving tumor cell survival and therapeutic resistance in breast cancers. In luminal breast cancers, ErbB3 upregulation following treatment with the anti-estrogen fulvestrant enhances PI3K/mTOR-mediated cell survival. However, the mechanism by which ErbB3 is upregulated in fulvestrant-treated cells is unknown. We found that ErbB3 protein levels and cell surface presentation were increased following fulvestrant treatment, focusing our attention on proteins that regulate ErbB3 at the cell surface, including Nrdp1, NEDD4, and LRIG1. Among these, only LRIG1 correlated positively with ERa, but inversely with ErbB3 in clinical breast cancer datasets. LRIG1, an estrogen-inducible ErbB down-regulator, was decreased in a panel of fulvestrant-treated luminal breast cancer cells. Ectopic LRIG1 expression from an estrogen-independent promoter uncoupled LRIG1 from estrogen regulation, thus sustaining LRIG1 and maintaining low ErbB3 levels in fulvestrant-treated cells. An LRIG1 mutant lacking the ErbB3 interaction motif was insufficient to down-regulate ErbB3. Importantly, LRIG1 overexpression improved fulvestrant-mediated growth inhibition, while cells expressing the LRIG1 mutant were poorly sensitive to fulvestrant, despite effective ERa downregulation. Consistent with these results, LRIG1 expression correlated positively with increased disease-free survival in anti-estrogen-treated breast cancer patients. These data suggest that ERadependent expression of LRIG1 dampens ErbB3 signaling in luminal breast cancer cells, and by blocking ERa activity with fulvestrant, LRIG1 is decreased thus permitting ErbB3 accumulation, enhanced ErbB3 signaling to cell survival pathways, and blunting therapeutic response to fulvestrant.

\footnotetext{
Users may view, print, copy, and download text and data-mine the content in such documents, for the purposes of academic research, subject always to the full Conditions of use:http://www.nature.com/authors/editorial_policies/license.html\#terms

${ }^{*}$ To whom correspondence should be addressed: Rebecca S. Cook, Vanderbilt University School of Medicine, Vanderbilt Ingram Cancer Center, Department of Cancer Biology, 2220 Pierce Ave., 759 PRB, Nashville, TN 37232, Phone: 615-936-3813, ; Email: rebecca.cook@vanderbilt.edu

Conflicts of interest. The authors have no conflicts of interest to declare.

Conflict of Interest Declaration. The Authors declare no competing financial interests in relation to the work described, and declare no conflict of interest.
} 


\section{Keywords}

Breast cancer; ErbB3; estrogen; LRIG; phosphatidyl inositol 3 kinase

\section{Introduction}

$E R B B 3$, the gene encoding the ErbB receptor tyrosine kinase (RTK) family member ErbB3, is expressed at relatively high levels in breast cancers that are $H E R 2$-amplified ${ }^{1}$, and at even higher levels in Luminal A breast cancers ${ }^{2}$, which often express and are dependent on estrogen receptor (ER)-a. Nearly $20 \%$ of all Luminal breast tumors exhibit ERBB3 gene copy number gains, causing increased $E R B B 3$ expression, and correlating with decreased disease-free survival ${ }^{2}$. ErbB3 expression is increased further in luminal breast cancers in response to treatment with the selective ERa modulator (SERM) tamoxifen or the selective ERa down-regulator (SERD) fulvestrant ${ }^{3}{ }^{3}$, two drugs clinically prescribed to patients with luminal breast cancer. Little is known regarding the role of ErbB3 in luminal breast tumors, largely due to a historical description of ErbB3 as a kinase-impaired heterodimeric partner of EGFR and ErbB2 $2^{16,},{ }^{6}$. However, increasing evidence suggest that ErbB3 is a key therapeutic target in luminal breast cancers.

Because ErbB3 harbors six phospho-tyrosine motifs that bind to the p85 regulatory subunit of phosphatidyl inositol-3-kinase (PI3K) ${ }^{1,8}{ }_{-}^{11}$, ErbB3 is a potent activator of PI3K signaling ${ }^{12}$. Once active, PI3K generates PIP3, a potent second messenger that recruits PI3K pathway effectors to the plasma membrane (e.g., serine-threonine kinases PDK-1 and Akt), resulting in their activation. Since Akt sits atop a signaling cascade that enhances cell growth and survival ${ }^{13}$, ErbB3 signaling promotes survival of luminal breast cancer cells. Thus, ErbB3 up-regulation in response to tamoxifen or fulvestrant increases PI3K-Akt signaling within 24 hours of treatment, increasing tumor cell survival and blunting the therapeutic benefit of these endocrine inhibitors $2,,, 9$, . However, combination of endocrine inhibitors with strategies designed to target ErbB3 may be used to circumvent the compensatory up-regulation of ErbB3-PI3K-Akt signaling, and improve luminal breast cancer cell killing. The therapeutic ErbB3 antibody U3-1287, for example, significantly impaired PI3K signaling and survival in fulvestrant-treated luminal breast tumor cells ${ }^{2}$.

The mechanism(s) contributing to ErbB3 up-regulation in response to fulvestrant are poorly understood. However, recent findings demonstrated a 2-fold up-regulation of ERBB3 transcripts in a panel of luminal breast cancer cells $24 \mathrm{~h}$ after fulvestrant treatment ${ }^{2}$. Thus, it is likely that additional mechanisms of ErbB3 regulation contribute to enhanced ErbB3 protein expression in fulvestrant-treated luminal breast cancer cells. We found that estrogendependent expression of the ErbB family regulatory factor LRIG1 maintains ErbB3 expression at low levels under basal conditions, and that fulvestrant disrupts ERa-dependent LRIG1 expression, permitting ErbB3 accumulation and survival signaling in fulvestranttreated cells. 


\section{Results}

\section{The ErbB3 antibody A4 neutralizes fulvestrant-mediated ErbB3 upregulation}

Previous studies demonstrate that humanized anti-ErbB3 monoclonal antibody A4 binds the ErbB3 ectodomain and effectively down-regulates ErbB3 in HER2-amplified breast cancer cells through receptor internalization ${ }^{14}, 15$. However, the utility of A4 in luminal breast cancers has not yet been described. We tested the impact of A4 on ErbB3 protein levels in two luminal breast cancer cell lines, MCF7 and T47D cells. Western analysis confirmed ErbB3 expression in serum-starved MCF7 and T47D cells (Fig. 1A), but showed that ErbB3 tyrosine phosphorylation at Y1289 was not detected under these conditions. Recombinant heregulin a1 (HRG, 2 ng/ml) induced ErbB3 Y1289 phosphorylation in control cells treated with non-specific human IgG. In contrast, pre-treatment of cells with A4 for $24 \mathrm{~h}$ prior to addition of HRG diminished HRG-induced ErbB3 phosphorylation at Y1289 in MCF7 and T47D, and decreased total ErbB3 levels.

Luminal breast cancer cells use ErbB3 up-regulation in response to ERa targeting to increase tumor cell growth and survival ${ }^{2},{ }^{8}, 9$. Therefore, we tested the ability of antibody A4 to neutralize ErbB3 up-regulation in luminal breast cancer cells treated with the selective ERa down-regulator (SERD) fulvestrant. MCF7 and T47D cells were treated for $24 \mathrm{~h}$ with fulvestrant in the presence or absence of A4. As expected, fulvestrant reduced expression of ERa, while upregulating expression of ErbB3 in control IgG-treated cultures (Fig. 1B), consistent with previous findings ${ }^{2}, 3$. However, treatment of luminal breast cancer cells with antibody A4 in combination with fulvestrant neutralized fulvestrant-mediated ErbB3 upregulation. In MCF7 and T47D cells treated with the combination of antibody A4 and fulvestrant, phosphorylation of Akt and ribosomal protein S6 was down-regulated to a greater extent that what was seen in cells treated with either agent alone.

To determine the impact of A4 on growth of luminal breast cancer cells, we treated $1 \times 10^{6}$ MCF7 and T47D cells with A4 for 3 days in media containing 10\% serum. Treatment of cells with A4 resulted in a modest but significant decrease in cell number after 3 days of treatment (Fig. 1C), consistent with previous reports using another ErbB3-specific antibody U3-1287 ${ }^{2}$. Treatment of cells with fulvestrant for 3 days decreased cell number. However, the combination of A4 with fulvestrant reduced cell number to a greater extent than either fulvestrant or A4 alone, confirming that ErbB3 up-regulation in fulvestrant-treated cells limits fulvestrant-mediated growth inhibition, and that targeting ErbB3 using the ErbB3 antibody A4 works in combination with fulvestrant to inhibit tumor cell growth through inhibition of ErbB3 expression. We investigated how ErbB3 targeting with antibody A4 affects the formation of fulvestrant-resistant colonies by seeding MCF7 and T47D cells at low density. Cells were cultured for 14 days in the presence of fulvestrant $(1 \mu \mathrm{M})$ or DMSO. Fulvestrant profoundly reduced colony formation in MCF7 and T47D cells (Fig. 1D). Antibody A4 also decreased colony formation in MCF7 and T47D cells as a single agent. However, the combination of fulvestrant + A4 caused superior growth inhibition as compared to fulvestrant alone or A4 alone. 


\section{Increased ErbB3 protein correlates with decreased ERa in luminal breast cancer cells}

Because fulvestrant down-regulates ERa while upregulating ErbB3 protein levels in cultured luminal breast cancer cell lines, we investigated the inverse relationship between ErbB3 and ERa in clinical luminal breast cancer specimens curated and analyzed by the Cancer Genome Atlas (TCGA, N = 321). We found high ErbB3 protein levels in 13.1\% (42 of 321) of tumors from the combined Luminal A and Luminal B subgroup as measured by reverse phase protein array (RPPA, Supp. Fig. S1A). Interestingly, the RPPA score for total ERa (ESR1) protein was significantly down-regulated in tumors with high ErbB3 protein levels (Fig. 2A), consistent with the inverse relationship between ERa and ErbB3 protein levels seen in cultured luminal breast cancer cells. Further, phosphorylation of ERa at S118 (which marks ERa transcriptional activity ${ }^{16}$ ) was substantially lower in luminal tumors with high ErbB3 protein levels (Supp. Fig. S1B). In contrast, ERBB3 transcripts were upregulated in $12 \%$ (37/324) luminal breast cancer specimens, but primarily in tumors distinct from those with ErbB3 protein up-regulation (Fig. 2B), demonstrating that ErbB3 mRNA may not predict ErbB3 protein levels in tumors. In contrast to the inverse relation observed between the RPPA scored for ErbB3 and ERa, no relationship was observed between ERa-Ser118 and $E R B B 3 \mathrm{mRNA}$ levels (Supp. Fig. S1C), while a positive relationship was seen between $E R B B 3 \mathrm{mRNA}$ and total ERa protein (Fig. 2A). These data suggest that ErbB3 protein and $E R R B 3 \mathrm{mRNA}$ correlate with ERa in distinct ways. This observation is consistent with the idea that ErbB3 protein expression, but not mRNA expression, is highest when ERa transcriptional activity is low.

We used avidin-mediated pull-down of biotinylated cell surface proteins followed by western analysis to determine if ErbB3 levels were upregulated at the cell surface of fulvestrant-treated MCF7 breast cancer cells. After treatment for 24 hours, ErbB3 pull-down increased more than 3-fold in fulvestrant treated cells as compared to cells treated with DMSO (Fig. 2C). ErbB3 was not pulled down by avidin beads in non-biotinylated cells, demonstrating the specificity of the assay. Thus ErbB3 protein presentation at the cell surface is upregulated in response to fulvestrant.

We further investigated fulvestrant-mediated ErbB3 up-regulation in four fulvestrant-treated luminal breast cancers cell lines, including lines with PIK3CA-H1047R activating mutations (T47D), PIK3CA-E545K activating mutations (MCF7 and MDA-MB-361), HER2 overexpression (MDA-MB-361) or with wild-type PIK3CA and HER2 (HCC1428). Fulvestrant caused ErbB3 up-regulation in each of these cell lines within 24 hours (Fig. 2D). Using cycloheximide (CHX) to block new protein synthesis, we found that ErbB3 protein levels decreased at time points following the addition of CHX to DMSO-treated cells, and were not detected after 120 minutes of CHX (Fig. 2D). In contrast, fulvestrant decreased ERa expression but increased ErbB3 expression over what was seen in DMSO-treated cells. In fulvestrant-treated HCC1428, MDA-MB-361 and T47D cells, ErbB3 remained abundant through 120 minutes after CHX treatment. In fulvestrant-treated MCF7 cells, however, ErbB3 diminished at a rate similar to what was seen in DMSO-treated cells. However, because baseline expression of ErbB3 was higher in fulvestrant-treated cells prior adding CHX, ErbB3 levels were still evident 120 minutes after CHX treatment. Thus, protein stabilization of ErbB3 may occur in response to fulvestrant in many luminal breast cancer 
cells, independently of PIK3CA mutation or HER 2 amplification, but other mechanisms may also lead to ErbB3 protein up-regulation in response to fulvestrant.

\section{LRIG1 decreases expression of ErbB3}

To understand the molecular events that increase cell surface ErbB3 protein levels in fulvestrant-treated luminal breast cancer cells, we turned our attention to proteins involved in ErbB3 trafficking and degradation, including LRIG1 ${ }^{17}, \mathrm{Nrdp}^{18}{ }^{18}{ }^{19}$, and NEDD4 ${ }^{20}$, which were over-expressed in 14\% (33/235), 9\% (22/235), and 8\% (19/235) of Luminal A breast cancers, respectively. RPPA data from TCGA-curated luminal breast cancers did not reveal a report for total ErbB3 levels in tumors queried for $L R I G 1$ or NRDP1 mRNA up-regulation, although total ErbB3 RPPA sores were reported upon query for $N E D D 4$ up-regulation, revealing no association between ErbB3 protein levels and NEDD4 transcript expression (Supp. Fig. S2). However, the RPPA score for phospho-ErbB3 Y1289, a phospho-tyrosine induced by treatment of breast cancer cells with HRG (Fig. 1A) or fulvestrant (Fig. 1B), was reported in queries for $L R I G 1, N r d p 1$, and NEDD4. Although no association was observed between P-ErbB3 Y1289 RPPA scores and either Nrdp1 or NEDD4, we found a statistically significant inverse relationship between LRIG1 and P-ErbB3 Y1289 (Fig. 3A). Therefore, we focused further attention on the relationship between LRIG1 and ErbB3 in luminal breast cancer cells treated with and without fulvestrant.

We stably expressed a myc-epitope tagged LRIG1 construct ${ }^{17}$ in MCF7, HCC1428, and T47D cells (Fig. 3B). In addition to wild-type (WT) LRIG1, we also expressed a myctagged LRIG1 deletion mutant lacking the large ErbB3 interaction motif in the LRIG1 ectodomain $\left(\mathrm{LRIG}^{\Delta \mathrm{Ecto}}{ }^{21}\right.$, and an LRIG1 mutant lacking the short regulatory/inhibitory cytoplasmic domain $\left(\mathrm{LRIG}^{\Delta \mathrm{Cyto}}\right)^{21}$. Cells were grown in $10 \%$ serum, and we assessed cell lysates by western analysis for ErbB3. We found that LRIG1 ${ }^{\mathrm{WT}}$ overexpression modestly decreased ErbB3 protein levels in each cell line as compared to cells expressing the empty vector pcDNA3, whereas expression of LRIG1 ${ }^{\Delta E c t o}$, which lacks the ErbB3 interaction motif, increased ErbB3 protein levels in each cell line tested. In contrast, ErbB3 was profoundly down-regulated in cells expressing LRIG1 $1^{\Delta \text { Cyto }}$, consistent with the reported increased ErbB-targeting activity of LRIG1 ${ }^{\Delta \text { Cyto }}$.

\section{LRIG1 inhibits growth of luminal breast cancer cells through ErbB3 down-regulation}

Previous studies demonstrate that LRIG1 suppresses growth of cancer cells ${ }^{22} 25$. We used Kaplan Meier Plotter (kmplot.com) to examine patient survival in datasets of tamoxifentreated $\mathrm{ER}+$ breast cancers $(\mathrm{N}=665)$, bifurcating these tumors into those expressing high or low levels of LRIG1. The patients in this combined dataset were treated with tamoxifen. Like fulvestrant, tamoxifen blocks the action of ERa, albeit through a distinct mechanism which warrants careful consideration in the context of the presented data. However, we used this publically available meta-analysis tool to identify potentially important information regarding an association between LRIG1 and response to endocrine-treated breast cancers. This approach identified a statistically significant decrease in disease-free survival for patients with tumors expressing lower LRIG1 levels (Fig. 4A). In contrast, expression of $N R D P 1$ or $N E D D 4$ did not correlate with disease-free survival of tamoxifen-treated ER+ breast cancer patients (Supp. Fig. S3). These data suggest that LRIG1 expression and 
activity promotes a favorable outcome following tamoxifen treatment, and conversely that lower LRIG1 levels may predict a worse outcome for tamoxifen-treated patients.

To study this more directly, and to determine the role of ErbB3 in LRIG1-mediated growth inhibition, we measured the impact of exogenous LRIG1 expression in luminal breast cancer cells. 2.0 X $10^{6} \mathrm{MCF} 7$ and HCC1428 cells stably expressing LRIG1 ${ }^{\mathrm{WT}}$, LRIG1 ${ }^{\Delta \mathrm{E} \text { ecto }}$, or the empty expression vector pcDNA3.1 were seeded and cultured with and without the ErbB3 antibody A4 for 3 days. Western analysis confirmed that ErbB3 levels were reduced upon A4 treatment in cells expressing the empty vector or expressing LRIG1 ${ }^{\triangle E c t o}$ as compared to these same cells treated with a control IgG (Fig. 4B). Although ErbB3 levels were already substantially reduced in cells expressing LRIG1 ${ }^{\text {WT }}$, ErbB3 protein levels were further reduced by A4. Cell counting revealed fewer cells in IgG-treated samples expressing LRIG1 ${ }^{\text {WT }}$ as compared to cells expressing the empty pcDNA3.1 vector, but increased cells in cultures expressing LRIG1 ${ }^{\Delta \text { Ecto }}$ (Fig. $4 \mathrm{C}$ ). Cell growth was substantially reduced in LRIG1 $^{\Delta E \text { Ecto }}$ cultures treated with antibody A4, suggesting that cells expressing LRIG1 ${ }^{\Delta E c t o}$ have a growth advantage over cells expressing the empty vector due in large part to expression of ErbB3. A4 did not further decrease the growth of cells expressing LRIG1 ${ }^{\mathrm{WT}}$. These results were confirmed using siRNA-mediated ErbB3 downregulation. MCF7 cells

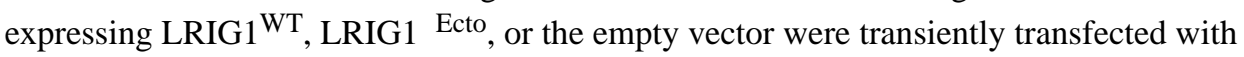
siRNA against ErbB3. At $24 \mathrm{~h}$ after transfection, $1.0 \times 10^{5}$ cells were seeded in 6-well dishes, and cultured for 3 days in 10\% serum, at which time parallel wells were assessed by western analysis or by cell counting. Western analysis of whole cell lysates confirmed decreased expression of ErbB3 in cells expressing LRIG1 ${ }^{\text {WT }}$, and increased ErbB3 levels in cells expressing LRIG1 ${ }^{\Delta \mathrm{Ecto}}$ as compared to cells harboring the empty vector. Cells transfected with siRNA sequences directed against ErbB3 demonstrated a reduction in ErbB3 levels (Fig. 4D). Cell counting revealed that knock-down of Erbb3 reduced the growth of MCF7 cells expressing LRIG1 ${ }^{\triangle E c t o}$ and MCF7 cells expressing the empty vector (Fig. 4E). Although cells expressing LRIG1 ${ }^{\mathrm{WT}}$ displayed reduced growth as compared to cells expressing empty pcDNA3.1, knockdown of ErbB3 did not decrease growth of these cells further. These observations are consistent with the idea that the tumor suppressor LRIG1 decreases growth of luminal breast cancer cells through down-regulation of ErbB3.

To understand the consequences of LRIG1 depletion, we used two distinct siRNA sequences directed against human LRIG1 to knock-down LRIG1 expression. Western analysis confirmed knockdown of LRIG1 following transient transfection of sequences targeting LRIG1, but not control siRNA sequences (Fig. 4F). ErbB3 levels were increased in MCF7 and HCC1428 cells upon LRIG1 knock-down, but were reduced in the presence of antibody A4. To assess the impact of LRIG1 knock-down on growth of luminal breast cancer cells, we seeded $2.0 \times 10^{6}$ MCF7 and HCC1428 cells 24 hours after siRNA transfection, then cultured cells for an additional 3 days in 10\% serum, with and without antibody A4. Cell counting after 3 days revealed increased growth of cells transfected with LRIG1 siRNA as compared to cells transfected with control siRNA sequences (Fig. 4G). Antibody A4 blocked the growth advantage conferred to MCF7 and HCC1428 cells by LRIG1 knockdown. Together, these data suggest that LRIG1 is a key mechanism restraining ErbB3mediated growth in luminal breast cells. 


\section{Decreased LRIG1 levels in fulvestrant-treated luminal breast cancer cells}

Previous reports demonstrate that estrogen-activated ERa transactivates the LRIG1 gene ${ }^{22}$. Consistent with these findings, Luminal breast cancers with high ERa RPPA scores displayed significantly higher expression of LRIG1 mRNA (Fig. 5A; $\mathrm{N}=235, \mathrm{P}=0.003$ ). In contrast, ERa RPPA scores did not correlate with Nrdp1 or NEDD4 expression in luminal breast cancers. These data are consistent with the idea that LRIG1 is an ERa-activated factor in luminal breast cancers ${ }^{22}$. Therefore, we tested the hypothesis that ERa down-regulation in response to fulvestrant causes decreased expression of LRIG1. LRIG1 down-regulation was seen in MCF7, MDA-MB-361, HCC1428, and T47D cells cultured $24 \mathrm{~h}$ with fulvestrant as compared to LRIG1 levels seen in DMSO-treated cells (Fig. 5B). Although down-regulation of ERa was nearly complete, LRIG1 down-regulation, as measured by western analysis, was partial in MCF7 and MDA-MB-361 cells, although LRIG1 was more substantially down-regulated in T47D and HCC1428 cells. We measured LRIG1 mRNA levels in MCF7, T47D, HCC1428, and MDA-MB-361 cells cultured $24 \mathrm{~h}$ in the presence or absence of fulvestrant, revealing a substantial reduction in LRIG1 mRNA levels in fulvestrant-treated cells (Fig. 5C). Further, MCF7 and MDA-MB-361 xenografts from mice treated with fulvestrant for 7 days $(\mathrm{N}=7)$ expressed a reduced level of $L R I G 1$ transcripts as compared to control-treated tumors (Fig. 5D). Conversely, MCF7 cells and T47D cells cultured in 10\% charcoal-stripped serum and treated with $20 \mathrm{pM} \mathrm{17 \beta -estradiol} \mathrm{for} 24 \mathrm{~h}$ upregulated LRIG1 protein levels while concurrently down-regulating ErbB3 protein levels (Fig. 5E). This suggests that ERa positively regulates LRIG1 transcript levels in luminal breast cancer cells, although additional mechanisms may contribute to total LRIG1 protein levels.

\section{Uncoupling LRIG1 from ERa sustains ErbB3 down-regulation in fulvestrant-treated breast cancer cells}

To determine if exogenous expression of LRIG1 ${ }^{\mathrm{WT}}$ could maintain ErbB3 protein downregulation in fulvestrant-treated cells, we treated MCF7 cells expressing LRIG1 ${ }^{\text {WT }}$ with fulvestrant for $24 \mathrm{~h}$. Although LRIG1 levels were decreased in vector-control cells treated with fulvestrant, MCF7-LRIG1 ${ }^{\text {WT }}$ cells maintained high LRIG1 expression levels following fulvestrant treatment (Fig. 6A). Importantly, ErbB3 levels, which were elevated in fulvestrant-treated MCF7-pcDNA3 cells, remained low in fulvestrant-treated MCF7LRIG1 ${ }^{\text {WT }}$ cells. We also used biotinylation of cell surface proteins to demonstrate that fulvestrant-mediated up-regulation of cell surface ErbB3 is blocked in in cells expressing LRIG1WT as compared to MCF7-pcDNA3 cells (Fig. 6B). Because LRIG1 inhibits growth of luminal breast cancer cells, and fulvestrant decreases expression of LRIG1, we wanted to test the hypothesis that LRIG1 down-regulation in response to fulvestrant results in a growth advantage that partially masks the tumor cell growth inhibition that could be achieved by fulvestrant. We tested this hypothesis in fulvestrant-treated MCF7 cells. Fulvestrant decreased the growth of MCF7-pcDNA3 cells over 7 days (Fig. 6C). However, fulvestrantmediated growth inhibition was significantly greater in MCF7-LRIGWT cells and MCF7LRIG $^{\Delta \text { Cyto }}$ cells as compared to MCF7-pcDNA3.1 cells. In contrast, MCF7-LRIG1 ${ }^{\Delta E c t o}$ cells were relatively resistant to the growth inhibitory effects of fulvestrant, indicating that the ectodomain of LRIG1 is necessary for LRIG1-mediated growth inhibition. These data suggest that sustained expression of LRIG1 improves the response of luminal breast cancer 
cells to fulvestrant, and conversely that loss of ERa-dependent LRIG1 expression weakens tumor growth inhibition in response to fulvestrant. Western analysis confirmed continued up-regulation of ErbB3 at 7 days of fulvestrant treatment in MCF7 cells expressing pcDNA3 (Fig. 6D). Taken together, these data suggest that ERa drives expression of the growth inhibitory protein LRIG1, a protein necessary for ErbB3 down-regulation (Fig. 6C). Upon loss of ERa and hence loss of LRIG1, ErbB3 levels accumulate at the cell surface, contributing to growth in fulvestrant treated cells.

\section{Discussion}

We have shown previously that ErbB3 drives growth and survival of the normal luminal mammary epithelium through MAPK and PI3K pathways, respectively ${ }^{26}$. Similarly, luminal breast cancers also use ErbB3 to increase cell survival in a PI3K-dependent manner ${ }^{27}$, particularly in fulvestrant-treated cells ${ }^{2,3}$, making the combination of therapeutic ErbB3 antibodies with fulvestrant an attractive approach to treat luminal breast cancers.

Several reports suggest that genetically enforced ErbB3 overexpression enhances endocrine resistance in luminal breast cancer cells ${ }^{2,3}$. More recently, it was shown that ErbB3 upregulation in response to loss of ER activity is not a genetic event acquired over time, but instead is a mechanism inherent to many luminal breast cancer cells ${ }^{2}$. We demonstrate herein that ERa-dependent expression of LRIG1 $1^{22,28}$, a cell surface protein involved in ErbB family RTK trafficking and/or degradation ${ }^{17},{ }^{25}, 29$, is down-regulated in fulvestranttreated luminal breast cancer cells, allowing ErbB3 to accumulate on the surface of breast cancer cells (Fig. 2), where it sustains cell growth. These data are consistent with previous reports demonstrating that estrogen-activated ERa controls expression of LRIG1, such that estrogen deprivation or ERa targeting reduce expression of LRIG1 in luminal breast cancer cells $^{22}$. These findings explain the relationships shown here between ErbB3 protein (but not mRNA), ERa protein, and LRIG1 mRNA in luminal breast cancers.

Importantly, decreased $L R I G 1$ transcript levels correlated with decreased disease-free survival in tamoxifen-treated luminal breast cancers (Fig. 5). Because LRIG1 negatively regulates cell surface levels of ErbB family RTKs, it is possible that increased signaling through ErbB family RTKs contributes to the worse outcome for those luminal breast tumors with low LRIG1. The ErbB RTK family is comprised of four members, including epidermal growth factor receptor (EGFR), ErbB2, ErbB3 and ErbB $4{ }^{1,}{ }^{30}$. Although our studies focus on ErbB3, previous studies demonstrate that enforced over-expression of EGFR, ErbB2, or certain ErbB4 splice variants increases cell growth and survival in tamoxifen-treated luminal breast cancer cells ${ }^{3} 5,8,31$. While we have not ruled out the potential roles of EGFR, ErbB2, and ErbB4 in regulating the response of luminal breast tumors to fulvestrant, we found that antibody-based ErbB3 targeting neutralized ErbB3 up-regulation in fulvestrant-treated cells, resulting in profound growth inhibition. Blockade of post-therapeutic ErbB3 up-regulation by antibody A4 has been reported in Raf/Mek inhibitor-treated melanomas ${ }^{15,32}$, and EGFR inhibitor treated non-small cell lung cancers ${ }^{33}$. Moreover, we found that the ErbB3 antibody blocked cell growth caused by LRIG1 knock-down or dominant-negative LRIG1 ${ }^{\Delta E c t o}$ expression. 
In summary, we have shown herein that the ERa-regulated gene LRIG1 suppresses cell surface ErbB3 expression and signaling in luminal breast cancers, such that ErbB3 rapidly accumulates on the cell membrane following treatment with fulvestrant. Previous studies demonstrate the profound impact of ErbB3 up-regulation on PI3K-Akt-mTOR signaling in fulvestrant-treated breast cancers ${ }^{2,3}$. Although mTOR inhibitors are approved in combination with endocrine therapy to prevent the consequences of this feedback inhibition $^{34}$, mTOR inhibitors are often poorly tolerated. Because ErbB3-targeting antibodies block resurgent signaling through the PI3K-Akt pathway in fulvestrant-treated cells $^{2}$, these data suggest that ErbB3 antibodies might be a therapeutic alternative for use in combination with endocrine inhibitors.

\section{Materials and Methods}

\section{Cell Culture}

All human breast cancer cell lines (MCF7, T47D, HCC1428, MDA-MB-361) were obtained from American Tissue Type Collection (Rockville, MD) and were cultured in Dulbecco's Modified Eagle's Medium (DMEM) supplemented with 10\% fetal bovine serum (FBS) (Atlanta Biologics) except where indicated as serum-free. Fulvestrant $(1 \mu \mathrm{M})$ (SigmaAldrich) and Heregulin-1 $\beta$ epidermal growth factor-like domain $(2 \mathrm{ng} / \mathrm{ml}$, R\&D Systems), and the humanized ErbB3 monoclonal antibody A4 ${ }^{14}, 15$ were used where indicated. Colony assays were seeded in 6-well dishes ( $10^{5}$ cells/dish), and cultured $14 \mathrm{~d}$ in DMEM/10\% FBS. Cells were fixed in formalin, stained with crystal violet, imaged on a flatbed scanner, converted to binary images, and quantified using Image $\mathrm{J}$ software. Cells $\left(1 \mathrm{X} 10^{5}\right)$ were transfected using Xfect Reagent (Clontech) according to manufacturer's directions with $2 \mu \mathrm{g}$ plasmid DNA: pcDNA3.1 (Life Technologies/InVitrogen), pcDNA3.1-LRIG1, pcDNA3.1LRIG1- $\Delta$ Ecto, and pcDNA3.1-LRIG1- $\Delta$ Cyto $^{21},{ }^{22}$. LRIG1 siRNA and non-specific siRNA sequences were purchased (Dharmacon) and used for transfection of cells with Dharmafect (Dharmacon) according to manufacturer's directions.

\section{Western blotting and immunoprecipitation}

Cells were homogenized in ice-cold lysis buffer [50 mM Tris pH 7.4, $100 \mathrm{mM} \mathrm{NaF}, 120 \mathrm{mM}$ $\mathrm{NaCl}, 0.5 \% \mathrm{NP}-40,100 \mu \mathrm{M} \mathrm{Na}_{3} \mathrm{VO}_{4}, 1 \mathrm{X}$ protease inhibitor cocktail (Roche)]. $1 \mathrm{mg}$ lysates were used for immunorecipitation (IP) with ErbB3 (Santa Cruz Biotechnologies, C-17) or 9E10 (Sigma-Aldrich) at 1:200 (v/v) for $2 \mathrm{~h}$, then pulled down with Protein A/G+ agarose (Santa Cruz Biotechnologies) at 1:20. IPs or cell lyates $(20 \mu \mathrm{g})$ were denatured in reducing sample buffer, resolved by 4-12\% SDS-PAGE (Novex, Life Technologies), and transferred to nitrocellulose membranes (iBlot, Life Technologies). Membranes were blocked and probed with antibody as previously described ${ }^{26}$ using the following antibodies: ErbB3 (C-17, Santa Cruz Biotechnology; 1:1000); Y1289 P-ErbB3 (Cell Signaling; 1:1000); 9E10 (Sigma-Aldrich; 1:2000); LRIG1 (Sigma-Aldrich; 1:500), a-actin (Sigma-Aldrich; 1:20,000).

\section{Biotinylation of cell surface proteins}

Cells were cultured to $80-90 \%$ confluence, then incubated $30 \mathrm{~min}$ at $4^{\circ} \mathrm{C}$ with EZ-Link Sulfo-NHS-SS-Biotin in the Pierce Cell Surface Protein Isolation Kit (Thermo Scientific) 
according to manufacturer's specifications. Cells were lysed in Pierce Lysis Buffer (Thermo Scientific) supplemented with protease inhibitor cocktail (Roche), sonicated five times at low power on ice for 1 second each, and clarified by centrifugation. Cells were collected, washed, and eluted with dithiothreitol using NeutrAvidin Agarose beads provided with the Pierce Cell Surface Protein Isolation Kit (Thermo Scientific) according manufacturer's directions. Eluates were analyzed by western analysis.

\section{Human breast tumor gene expression analysis}

The Cancer Genome Atlas ${ }^{35}$ Invasive Breast Cancer dataset was clustered according to PAM50 subtypes and corresponding data on ERBB3, LRIG1, NEDD4, and NRDP1 mRNA, patient survival, and ErbB3, ErbB3 PY1289, ESR1, and ESR1 P-S118 were analyzed using publically available software on the cBio Portal website ${ }^{36}$. Meta-analysis of expression array datasets of tamoxifen-treated Luminal A breast cancers were assessed for correlations between disease-free survival and expression of LRIG1 (211596_s_at), NRDP1 (201961_s_at), and NEDD4 (213012_at) using KmPlotter (KmPlot.com).

\section{RNA extraction and quantitative reverse transcription-polymerase chain reaction (RT-PCR)}

Using RNeasy (Qiagen), total RNA was harvested from cell lysates passed through a QiaShredder column (Qiagen) or from flash-frozen tumors homogenized using a tissue lyser. Total RNA was quantified and 2 ug RNA was used to synthesize cDNA (High Capacity cDNA Reverse Transcription kit - Applied Biosystems). Quantitative RT PCR was performed on a BioRad iCycler iQ5 machine using the following primer sequences:

Human LRIG1 F: 5'-TAA CTC GAG AAC CCA CTG CTT ACT- 3'

Human LRIG1 R: 5'-CTA GAA GGC ACA CTC GAG GCT GAT- 3'

\section{Xenograft experiments}

Estrogen pellets ( $14 \mathrm{~d}$ extended release, $0.17 \mathrm{mg} \beta$ estradiol, Innovative Research of America) were implanted subcutaneouly into 3-4 week old female Balb/C athymic nude mice (Jackson Laboratories). Right and left inguinal mammary fatpads were injected with $10^{6}$ MCF7 or MDA-MB-361 in $100 \mu \mathrm{l}$ growth factor reduced Matrigel. Tumors were measured with calipers twice weekly. Tumor-bearing mice were randomized into treatment arms to receive fulvestrant (Novartis) delivered once weekly by intramuscular injection. All animal experimentation was performed in AAALAC approved facilities at Vanderbilt University Medical Center. All animal use protocols were reviewed and approved prior to experimentation by the Institutional Animal Care and Use Committee at Vanderbilt University, Nashville, TN.

\section{Statistics Overview}

To assess statistical significance in experiments directly comparing an experimental group to a control group, Student's unpaired, two-tail T-test was used. $\mathrm{P}<0.05$ was considered significantly different from the null hypothesis. 


\section{Supplementary Material}

Refer to Web version on PubMed Central for supplementary material.

\section{Acknowledgments}

Funding. This work was supported by the National Institutes of Health R01 CA143126 (RSC) and R01 CA118384 (CS), and Susan G. Komen for the Cure grant KG100677 (RSC).

\section{References}

1. Stern DF. ERBB3/HER3 and ERBB2/HER2 duet in mammary development and breast cancer. J Mammary Gland Biol Neoplasia. 2008; 13:215-223. [PubMed: 18454306]

2. Morrison MM, et al. ErbB3 downregulation enhances luminal breast tumor response to antiestrogens. J Clin Invest. 2013

3. Hutcheson IR, et al. Fulvestrant-induced expression of ErbB3 and ErbB4 receptors sensitizes oestrogen receptor-positive breast cancer cells to heregulin beta1. Breast Cancer Res. 2011; 13:R29. [PubMed: 21396094]

4. Tovey S, et al. Can molecular markers predict when to implement treatment with aromatase inhibitors in invasive breast cancer? Clin Cancer Res. 2005; 11:4835-4842. [PubMed: 16000581]

5. Liu B, et al. Downregulation of erbB3 abrogates erbB2-mediated tamoxifen resistance in breast cancer cells. Int J Cancer. 2007; 120:1874-1882. [PubMed: 17266042]

6. Kraus MH, Issing W, Miki T, Popescu NC, Aaronson SA. Isolation and characterization of ERBB3, a third member of the ERBB/epidermal growth factor receptor family: evidence for overexpression in a subset of human mammary tumors. Proc Natl Acad Sci U S A. 1989; 86:9193-9197. [PubMed: 2687875]

7. Kim HH, Vijapurkar U, Hellyer NJ, Bravo D, Koland JG. Signal transduction by epidermal growth factor and heregulin via the kinase-deficient ErbB3 protein. Biochem J. 1998; 334 ( Pt 1):189-195. [PubMed: 9693119]

8. Hamburger AW. The role of ErbB3 and its binding partners in breast cancer progression and resistance to hormone and tyrosine kinase directed therapies. J Mammary Gland Biol Neoplasia. 2008; 13:225-233. [PubMed: 18425425]

9. Sutherland RL. Endocrine resistance in breast cancer: new roles for ErbB3 and ErbB4. Breast Cancer Res. 2011; 13:106. [PubMed: 21639949]

10. Hellyer NJ, Cheng K, Koland JG. ErbB3 (HER3) interaction with the p85 regulatory subunit of phosphoinositide 3-kinase. Biochem J. 1998; 333 ( Pt 3):757-763. [PubMed: 9677338]

11. Kim HH, Sierke SL, Koland JG. Epidermal growth factor-dependent association of phosphatidylinositol 3-kinase with the erbB3 gene product. J Biol Chem. 1994; 269:24747-24755. [PubMed: 7929151]

12. Klempner SJ, Myers AP, Cantley LC. What a tangled web we weave: emerging resistance mechanisms to inhibition of the phosphoinositide 3-kinase pathway. Cancer Discov. 2013; 3:13451354. [PubMed: 24265156]

13. Wong KK, Engelman JA, Cantley LC. Targeting the PI3K signaling pathway in cancer. Curr Opin Genet Dev. 2010; 20:87-90. [PubMed: 20006486]

14. Aurisicchio L, et al. Novel anti-ErbB3 monoclonal antibodies show therapeutic efficacy in xenografted and spontaneous mouse tumors. J Cell Physiol. 2012; 227:3381-3388. [PubMed: 22213458]

15. Belleudi F, et al. Monoclonal antibody-induced ErbB3 receptor internalization and degradation inhibits growth and migration of human melanoma cells. Cell Cycle. 2012; 11:1455-1467. [PubMed: 22421160]

16. Ali S, Metzger D, Bornert JM, Chambon P. Modulation of transcriptional activation by liganddependent phosphorylation of the human oestrogen receptor A/B region. EMBO J. 1993; 12:11531160. [PubMed: 8458328] 
17. Laederich MB, et al. The leucine-rich repeat protein LRIG1 is a negative regulator of ErbB family receptor tyrosine kinases. J Biol Chem. 2004; 279:47050-47056. [PubMed: 15345710]

18. Diamonti AJ, et al. An RBCC protein implicated in maintenance of steady-state neuregulin receptor levels. Proc Natl Acad Sci U S A. 2002; 99:2866-2871. [PubMed: 11867753]

19. Qiu XB, Goldberg AL. Nrdp1/FLRF is a ubiquitin ligase promoting ubiquitination and degradation of the epidermal growth factor receptor family member, ErbB3. Proc Natl Acad Sci U S A. 2002; 99:14843-14848. [PubMed: 12411582]

20. Huang Z, et al. The E3 ubiquitin ligase NEDD4 negatively regulates HER3/ErbB3 level and signaling. Oncogene. 2014

21. Rafidi H, et al. Leucine-rich repeat and immunoglobulin domain-containing protein-1 (Lrig1) negative regulatory action toward ErbB receptor tyrosine kinases is opposed by leucine-rich repeat and immunoglobulin domain-containing protein 3 (Lrig3). J Biol Chem. 2013; 288:21593-21605. [PubMed: 23723069]

22. Krig SR, et al. Lrig1 is an estrogen-regulated growth suppressor and correlates with longer relapsefree survival in ERalpha-positive breast cancer. Mol Cancer Res. 2011; 9:1406-1417. [PubMed: 21821674]

23. Miller JK, et al. Suppression of the negative regulator LRIG1 contributes to ErbB2 overexpression in breast cancer. Cancer Res. 2008; 68:8286-8294. [PubMed: 18922900]

24. Shattuck DL, et al. LRIG1 is a novel negative regulator of the Met receptor and opposes Met and Her2 synergy. Mol Cell Biol. 2007; 27:1934-1946. [PubMed: 17178829]

25. Stutz MA, Shattuck DL, Laederich MB, Carraway KL 3rd, Sweeney C. LRIG1 negatively regulates the oncogenic EGF receptor mutant EGFRvIII. Oncogene. 2008; 27:5741-5752. [PubMed: 18542056]

26. Balko JM, et al. The receptor tyrosine kinase ErbB3 maintains the balance between luminal and basal breast epithelium. Proceedings of the National Academy of Sciences of the United States of America. 2012; 109:221-226. [PubMed: 22178756]

27. Cook RS, et al. ErbB3 ablation impairs PI3K/Akt-dependent mammary tumorigenesis. Cancer Res. 2011; 71:3941-3951. [PubMed: 21482676]

28. Ghasimi S, et al. Immunohistochemical analysis of LRIG proteins in meningiomas: correlation between estrogen receptor status and LRIG expression. J Neurooncol. 2012; 108:435-441. [PubMed: 22484910]

29. Wong VW, et al. Lrig1 controls intestinal stem-cell homeostasis by negative regulation of ErbB signalling. Nat Cell Biol. 2012; 14:401-408. [PubMed: 22388892]

30. Wiseman SM, et al. Coexpression of the type 1 growth factor receptor family members HER-1, HER-2, and HER-3 has a synergistic negative prognostic effect on breast carcinoma survival. Cancer. 2005; 103:1770-1777. [PubMed: 15770691]

31. Sundvall M, et al. Role of ErbB4 in breast cancer. J Mammary Gland Biol Neoplasia. 2008; 13:259-268. [PubMed: 18454307]

32. Fattore L, et al. Activation of an early feedback survival loop involving phospho-ErbB3 is a general response of melanoma cells to RAF/MEK inhibition and is abrogated by anti-ErbB3 antibodies. J Transl Med. 2013; 11:180. [PubMed: 23890105]

33. Noto A, et al. Combination therapy with anti-ErbB3 monoclonal antibodies and EGFR TKIs potently inhibits non-small cell lung cancer. Oncotarget. 2013; 4:1253-1265. [PubMed: 23896512]

34. Baselga J, et al. Everolimus in postmenopausal hormone-receptor-positive advanced breast cancer. N Engl J Med. 2012; 366:520-529. [PubMed: 22149876]

35. Comprehensive molecular portraits of human breast tumours. Nature. 2012; 490:61-70. [PubMed: 23000897]

36. Cerami E, et al. The cBio cancer genomics portal: an open platform for exploring multidimensional cancer genomics data. Cancer Discov. 2012; 2:401-404. [PubMed: 22588877] 
A

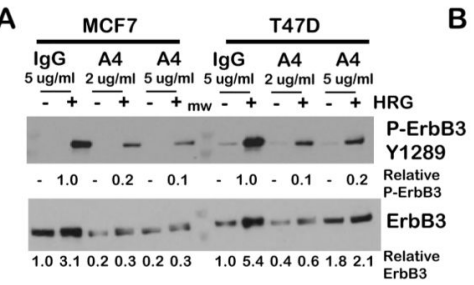

C

Cell growth over 72 hours

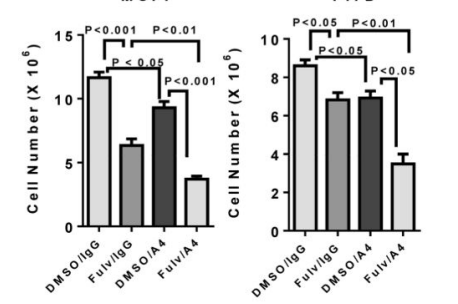

D DMSo Fulv.

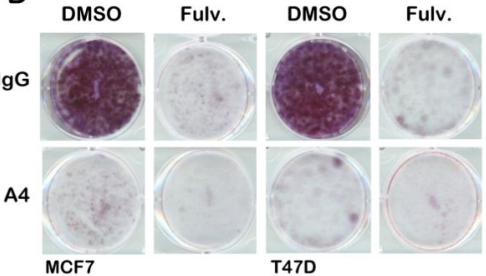

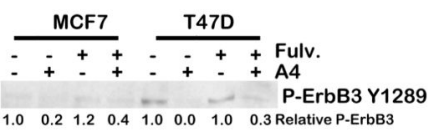

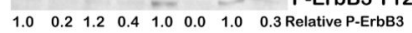

- $\quad-\quad-\infty-$ ErbB3

$\begin{array}{llllllll}1.0 & 0.3 & 4.8 & 0.1 & 1.0 & 0.1 & 2.3 & 0.6 \text { Relative ErbB3 }\end{array}$

- - ER - - ER

\begin{tabular}{llllllll}
1.0 & 1.0 & 0.5 & 0.5 & 1.0 & 1.2 & 0.2 & 0.1 \\
\hline
\end{tabular}

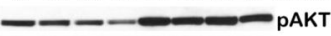

$\begin{array}{lllllllllll}1.0 & 0.7 & 0.5 & 0.3 & 1.0 & 0.9 & 1.0 & 0.8 & \text { AKT Relative P-Akt }\end{array}$

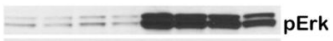

- = = - - - Erk

$\begin{array}{lllllllll}1.0 & 0.7 & 1.3 & 0.8 & 1.0 & 0.9 & 0.8 & 0.9 \text { Relative P-Erk }\end{array}$

- $-\quad$ pS6

- C

$\begin{array}{llllllll}1.0 & 1.1 & 0.3 & 0.1 & 1.0 & 2.2 & 0.3 & 0.0 \\ \end{array}$

-

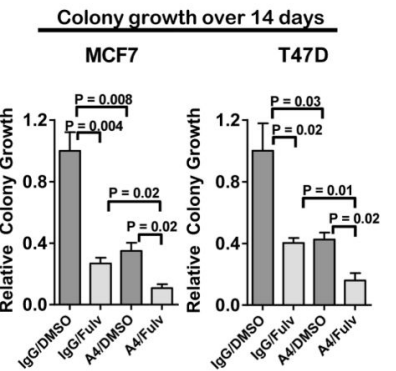

Figure 1. Fulvestrant-mediated ErbB3 up-regulation is neutralized by the ErbB3 antibody A4 A. Western analysis of whole cell lysates collected from serum-starved MCF7 and T47D cells treated $24 \mathrm{~h}$ with antibody A4 $(2-5 \mu \mathrm{g} / \mathrm{ml})$ or non-specific human $\mathrm{IgG}$, and for the final 10 min of culture with recombinant $\operatorname{HRG} \beta 1(2 \mathrm{pg} / \mathrm{ml})$.

B. Western analysis of whole cell lysates cultured in $10 \%$ serum and treated $24 \mathrm{~h}$ with fulvestrant $(1 \mu \mathrm{M})$ or antibody A4 $(2 \mu \mathrm{g} / \mathrm{ml})$.

C. Cells cultured for 3 days in $10 \%$ serum in the presence or absence of fulvestrant $(1 \mu \mathrm{M})$ or antibody A4 $(2 \mu \mathrm{g} / \mathrm{ml})$ were collected by trypsinization and counted. Average cell number $( \pm$ S.D.) is shown. $\mathrm{N}=5$, each counted in duplicate. Student's T-test.

D. Left panel: Representative images of crystal violet-stained cells after $14 \mathrm{~d}$ culture in $10 \%$ serum in the presence or absence of fulvestrant $(1 \mu \mathrm{M})$ and antibody A4 (2 $\mu \mathrm{g} / \mathrm{ml})$. Right panel: Crystal violet fluorescence was measured on the Odyssey scanner, and used as a relative measure of total cell number, setting the average fluorescence value for cells cultured in IgG/DMSO equal to 1 . Average \pm S.D. is shown. $\mathrm{N}=3$, each assessed in triplicate. Student's T-test. 


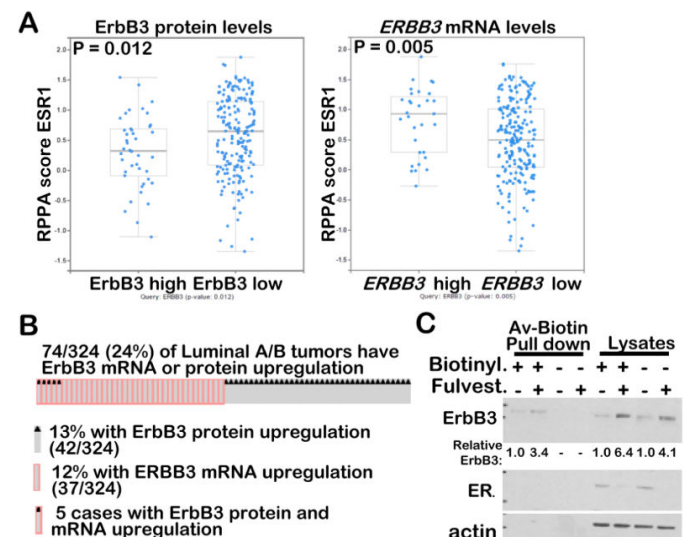

5 cases with ErbB3 protein and
mRNA upregulation

actin
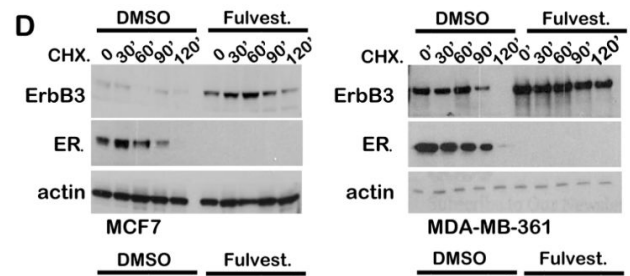

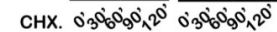
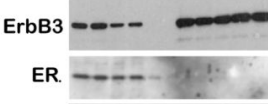

actin $=0.00-0.00$

HCC1428

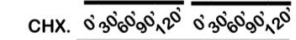

ErbB3

ER.

actin

$-\infty-\infty$

T47D

Figure 2. ErbB3 protein levels correlate inversely with ERa, but ERBB3 mRNA levels do not A. Using TCGA-curated luminal breast cancer datasets, the RPPA protein score for ESR1 (ERa) was plotted for those tumors with high ErbB3 protein expression or high ERBB3 mRNA expression as compared to the remaining luminal tumors. Midlines represent average, whiskers indicate S.D. Analysis was performed on cBioPortal.org.

B. Oncoprint generated using cBioportal software (www.cbioportal.org) to analyze TCGAcurated Luminal A/B tumors for tumors with high ErbB3 RPPA scores or high ERBB3 mRNA expression. Unaltered cases were removed to demonstrate the distinct compartments where ErbB3 mRNA and protein up-regulation fail to overlap.

C. Western analysis of whole cell lysates or avidin-biotin pull-downs from biotin-labelled MCF7 cells (lanes 1-2 and 5-6). Cells without biotin labeling were used as negative controls for avidin-biotin pull downs.

D. Western analysis of whole cell lysates harvested from cells cultured in $10 \%$ serum for 24 h with DMSO or fulvestrant $(1 \mu \mathrm{M})$, and for the final 0-120 minutes of culture with cycloheximide (CHX), 

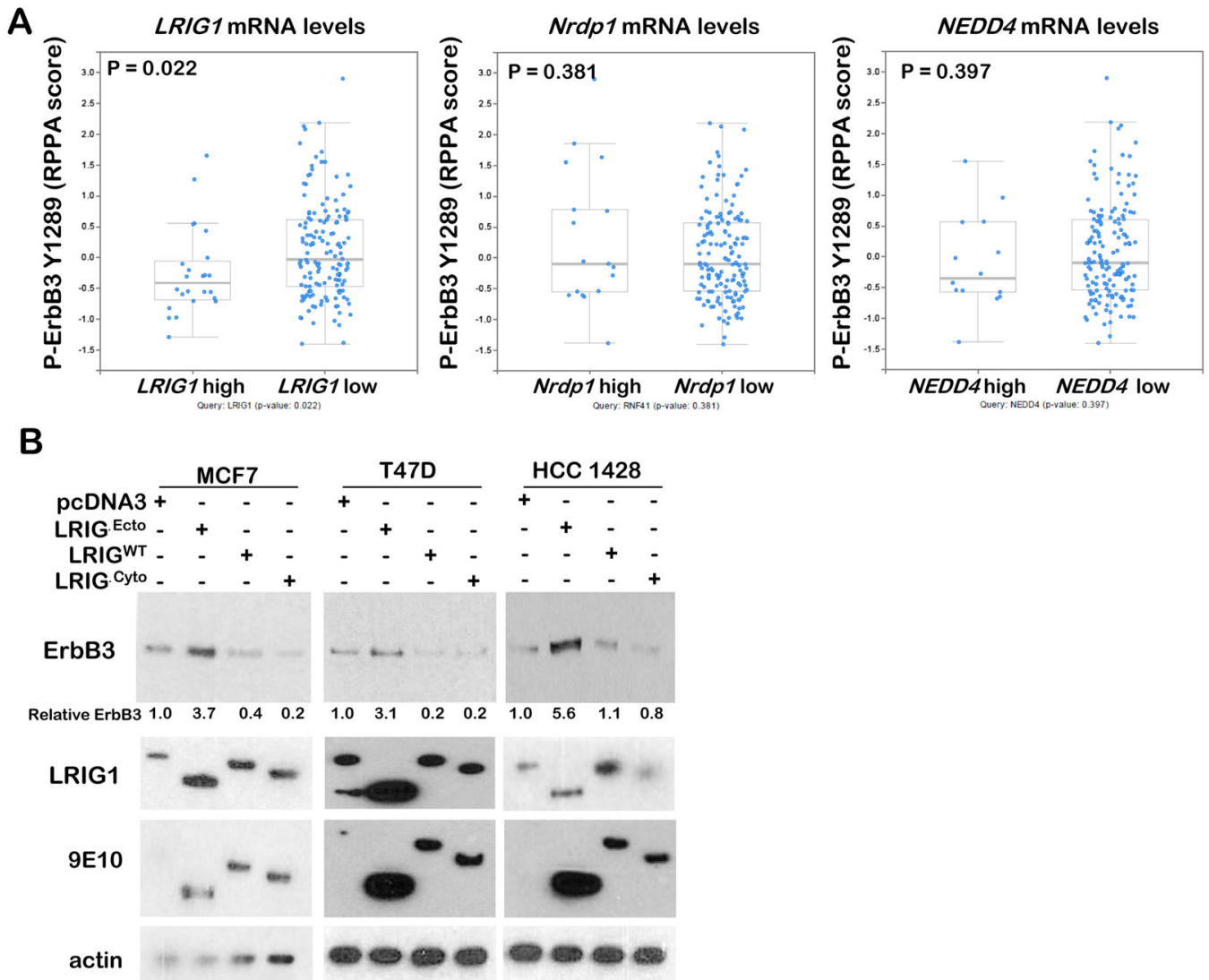

Figure 3. LRIG1 decreases expression of ErbB3

A. Using TCGA-curated luminal breast cancer datasets, the RPPA protein score for ErbB3 phospho-Tyr1289 was plotted for those tumors with high LRIG1, NRDP1, and NEDD4 mRNA expression as compared to the remaining luminal tumors. Midlines represent average, whiskers indicate S.D. Analysis was performed on cBioPortal.org.

B. Western analysis of whole cell lysates harvested from cells stably expressing the empty pcDNA3.1 vector, LRIG1 $\Delta$ Ecto (a dominant negative LRIG1 mutant), LRIG1-WT, and LRIG1 $\Delta$ Cyto (an active LRIG1 mutant). 
A

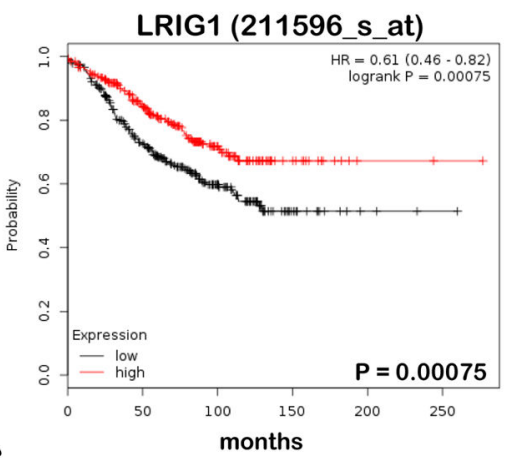

C

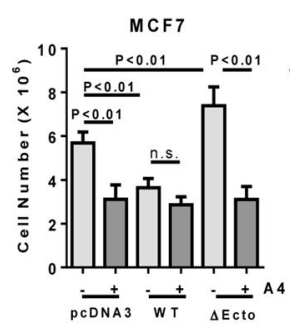

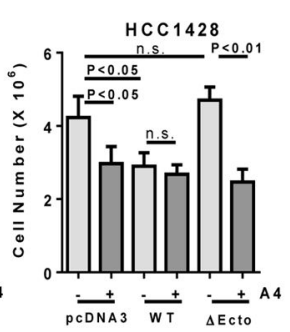

B

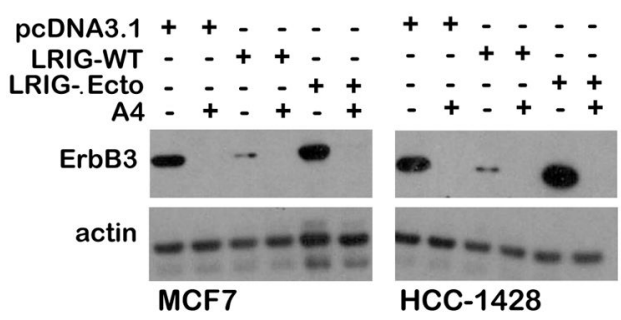

D

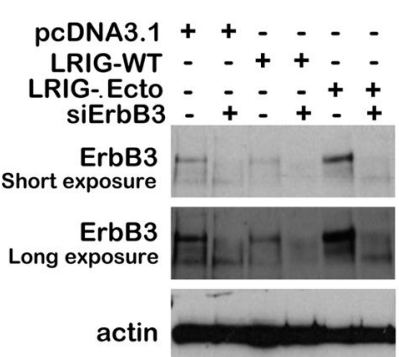

E

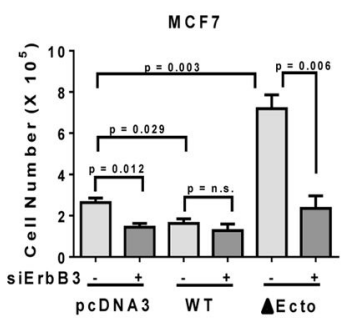

$\mathbf{F}$

Ctrl. siRNA + + - - + + - LRIG SIRNA.1 - - + + - - + + - -

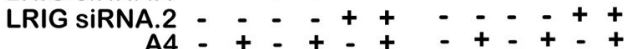

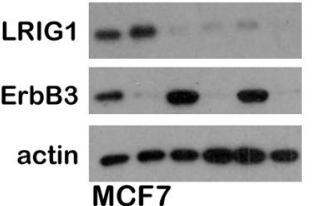
MCF7
G

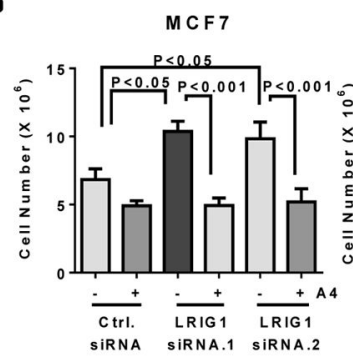

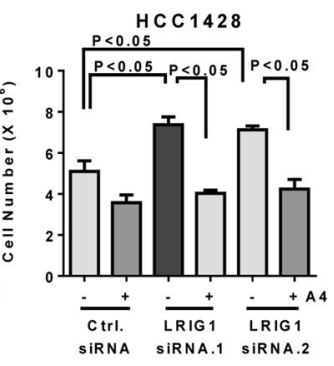

Figure 4. ErbB3 up-regulation drives increased growth of cells lacking LRIG1 activity

A. Transcript levels of $L R I G 1$ were assessed in tamoxifen-treated breast cancer expression microarray datasets and used to generate a Kaplan-Meier survival curve using KmPlot.com. Analysis included all tumors treated with or without chemotherapy, and excluded all samples not treated with tamoxifen. The probes used for analysis are indicated at top of panel.

B. Western analysis of whole cell lysates harvested form cells stably expressing pcDNA3.1, LRIG-WT, or LRIG1- $\Delta$ Ecto, and cultured 24 hours in $10 \%$ serum with antibody A4 (2 $\mu \mathrm{g} / \mathrm{ml}$ ) or IgG.

C. Cells cultured for 3 days in $10 \%$ serum in the presence or absence of antibody A4 (2 $\mu \mathrm{g} / \mathrm{ml}$ ) were collected by trypsinization and counted. Average cell number ( \pm S.D.) is shown. $\mathrm{N}=3$, each counted in duplicate. Student's T-test.

D. Western analysis of whole cell lysates harvested form cells transiently transfected with ErbB3 siRNA sequences or with non-targeting siRNA sequences. $24 \mathrm{~h}$ after transfection cells were seeded, and cultured 72 hours in $10 \%$ serum prior to collection for western analysis.

E. 24 hours after transient transfection with ErbB3 siRNA sequences or with non-targeting siRNA sequences, equal number of cells were seeded and cultured in $10 \%$ serum for $3 \mathrm{~d}$. 
Cells were collected by trypsinization and counted. Average cell number ( \pm S.D. $)$ is shown. $\mathrm{N}=5$, each counted in duplicate. Student's T-test.

F. Western analysis of whole cell lysates harvested from cells transiently transfected with LRIG1 siRNA sequences or non-targeting siRNA sequences. 72 hours after transfection, cells were cultured in $10 \%$ serum with antibody A4 $(2 \mu \mathrm{g} / \mathrm{ml})$ or IgG for $24 \mathrm{~h}$.

G. 24 hours after transient transfection with LRIG1 siRNA sequences or with non-targeting siRNA sequences, cells were cultured in $10 \%$ serum with antibody A4 $(2 \mu \mathrm{g} / \mathrm{ml})$ or IgG for $3 \mathrm{~d}$. Cells were collected by trypsinization and counted. Average cell number ( \pm S.D.) is shown. $\mathrm{N}=5$, each counted in duplicate. Student's T-test. 
A
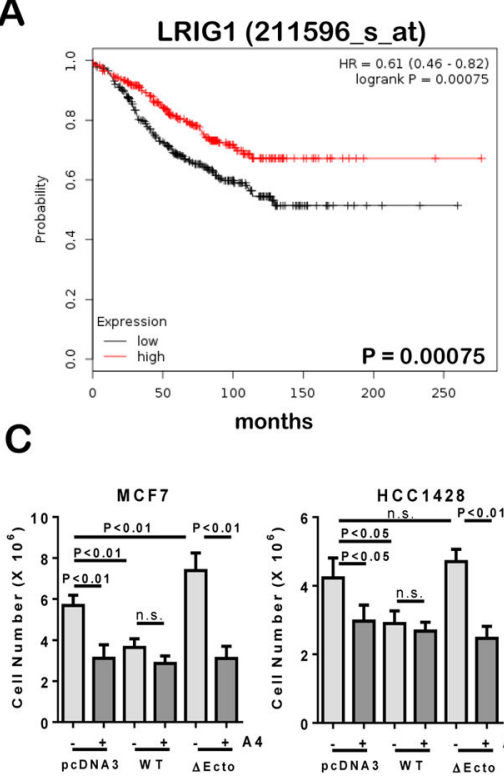

F

Ctrl. siRnA + + - - + + + - -

LRIG SiRNA.1 - - + + - - - + + - -

LRIG SIRNA.2 $-\div \div+ \pm+\div \div-+ \pm+$

LRIG1

ErbB3 - - -

actin

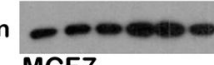

MCF7
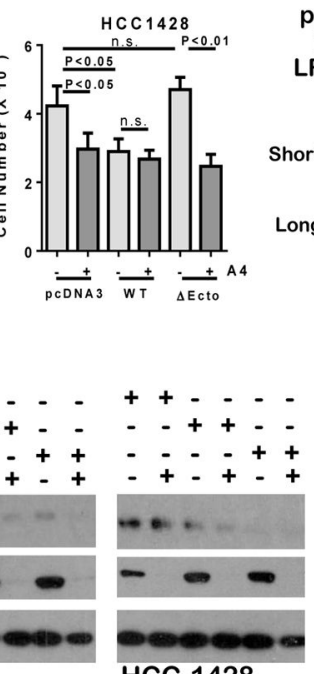

HCC-1428
D
B

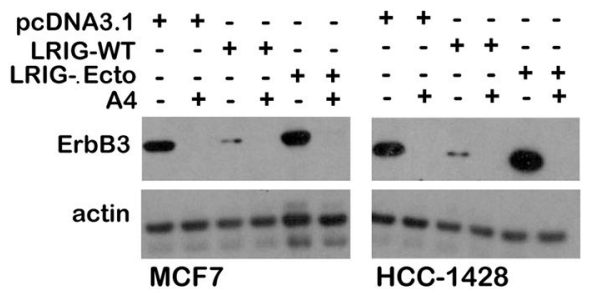

E

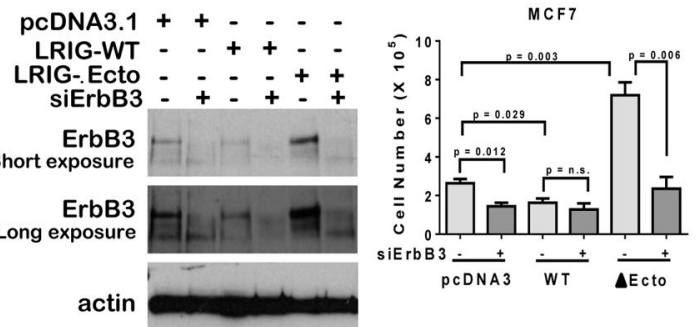

G
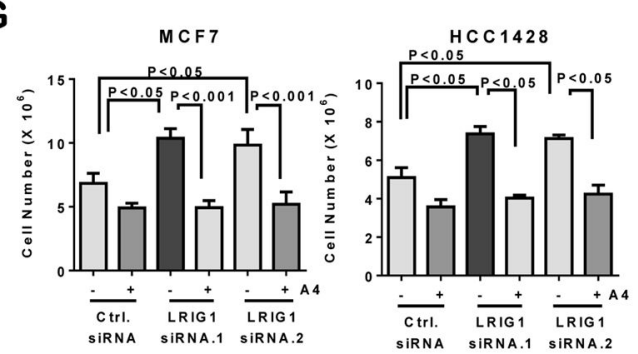

Figure 5. LRIG1 decreases growth of luminal breast cancer cells

A. Using TCGA-curated luminal breast cancer datasets, the RPPA protein score for ESR1 (ERa) was plotted for those tumors with high LRIG1, NRDP1, and NEDD4 mRNA expression as compared to the remaining luminal tumors. Midlines represent average, whiskers indicate S.D. Analysis was performed on cBioPortal.org.

B. Western analysis of whole cell lysates harvested form cells cultured $24 \mathrm{~h}$ in $10 \%$ serum with fulvestrant $(1 \mu \mathrm{M})$ or equal volume of DMSO.

C. LRIG1 transcripts were measured in whole cell RNA harvested from cells cultured $24 \mathrm{~h}$ in $10 \%$ serum with fulvestrant $(1 \mu \mathrm{M})$ or DMSO. Values represent average relative mRNA levels ( \pm S.D.), $N=3$, each analyzed in triplicate. Student's T-test.

D. LRIG1 transcripts were measured in whole tumor RNA harvested from MCF7 and MDAMB-361 xenografts treated for 7 days with fulvestrant or vehicle. Values represent average relative mRNA levels $( \pm$ S.D. $), N=4$, each analyzed in triplicate. Student's T-test.

E. Western analysis of whole cell lysates harvested form cells cultured $24 \mathrm{~h}$ in $10 \%$ charcoal stripped serum with estrogen $(20 \mathrm{pM})$ or equal volume ethanol. Values represent average relative mRNA levels $( \pm$ S.D.), $N=3$, each analyzed in triplicate. Student's T-test. 

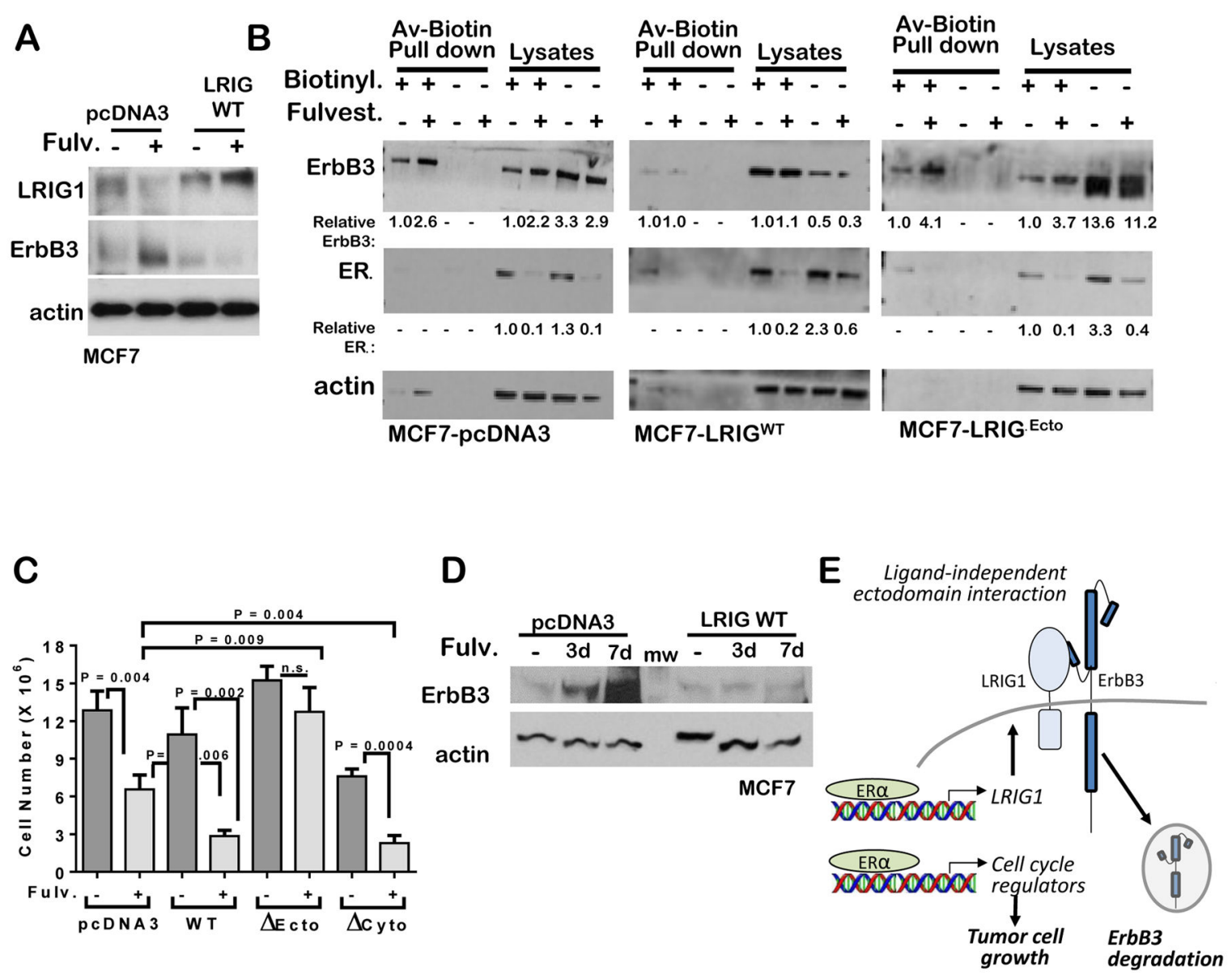

Figure 6. LRIG1 down-regulation drives fulvestrant-mediated ErbB3 up-regulation

A. Western analysis of whole cell lysates harvested form cells stably expressing pcDNA3.1 or LRIG1-WT. Cells cultured in $10 \%$ serum and with fulvestrant $(1 \mu \mathrm{M})$ or DMSO for $24 \mathrm{~h}$.

B. Western analysis of whole cell lysates or avidin-biotin pull-downs from cells labeled with or without biotin for 30 minutes following $24 \mathrm{~h}$ treatment with fulvestrant or DMSO.

C. Cells expressing pcDNA3, LRIG1-WT, LRIG1- $\Delta$ Ecto, or LRIG1- $\Delta$ Cyto were cultured in $10 \%$ serum with fulvestrant $(1 \mu \mathrm{M})$ or DMSO for $7 \mathrm{~d}$. Cells were collected by trypsinization and counted. Average cell number $( \pm$ S.D.) is shown. $\mathrm{N}=5$, each counted in duplicate.

Student's T-test.

D. Western analysis of whole cell lysates harvested form cells stably expressing pcDNA3.1 or LRIG1-WT. Cells cultured in $10 \%$ serum for 7 days, and with fulvestrant $(1 \mu \mathrm{M})$ for the final 3 days or 7 days, or cultured with DMSO for 7 days.

E. Schematic of how ERa-induced expression of LRIG1 maintains ErbB3 at low levels in luminal breast cancer cells. Endocrine inhibitors, such as fulvestrant, tamoxifen, or aromatase inhibitors would cause reduced LRIG1 expression levels, thus allowing ErbB3 accumulation at the cell surface. 